\title{
Assessing the cost of providing a prevention of mother-to-child transmission of HIV/AIDS service in Ethiopia: urban-rural health facilities setting
}

Elias Asfaw Zegeye ${ }^{1,2^{*}}$, Josue Mbonigaba ${ }^{1}$, Sylvia Kaye ${ }^{3}$ and Benjamin Johns ${ }^{4}$

\begin{abstract}
Background: While local context costing evidence is relevant for healthcare planning, budgeting and costeffectiveness analysis, it continues to be scarce in Ethiopia. This study assesses the cost of providing a prevention of mother-to-child transmission of HIV/AIDS (PMTCT) service across heterogeneous prevalence (high, low) and socioeconomic (urban, rural) contexts.

Methods: A total of 12 health facilities from six regions in Ethiopia were purposively selected from the latest 2012 antenatal sentinel HIV prevalence report. Six health facilities with the highest HIV prevalence (8.1 to 17.3\%) in urban settings and six health facilities with the lowest prevalence (0.0 to $0.1 \%)$ in rural settings were selected. A microcosting approach was applied to identify, measure and value resources used for the provision of a comprehensive PMTCT service. The analysis was conducted across different PMTCT service packages. We also estimated national costs in urban and rural contexts.

Results: The average cost per pregnant woman-infant pair per year (PPY) ranged from ETB 6280 (USD 319) to ETB 21,620 (USD 1099) in the urban high HIV prevalence health facilities setting. In rural low HIV prevalence health facilities, the cost ranged from ETB 4323 (USD 220) to ETB 7539 (USD 383).PMTCT service provision in urban health facilities costs more than twice the cost in rural health facilities. The average cost per PPY in an urban setting was more than double the cost in a rural setting due to the higher cost of inputs and possible inefficiencies (although there were a higher number of visits). Consumables (including antiretroviral drugs) and infrastructure were the major cost drivers in both the urban and rural health facilities. Among PMTCT service components, anti-retroviral treatment Option B+ follow-up and counselling accounted for the highest proportion of costs, which ranged from 58 to $72 \%$. Nationally, at the current coverage, the cost of PMTCT service was USD 6 million and USD 3 million in urban and rural settings, respectively.

Conclusions: The analysis suggests that resources used for PMTCT service packages varied across health facilities and HIV prevalence contexts. Providing PMTCT service in the high HIV prevalence urban health facilities costs more than in the rural facilities. Context-specific costing was vital to provide locally sensitive evidence for health service management and priority setting.
\end{abstract}

Keywords: Cost analysis, Unit cost, PMTCT service packages, Resource ingredients, Micro-costing, Urban, Rural and Ethiopia

\footnotetext{
* Correspondence: eliasasfawe@gmail.com

${ }^{1}$ Economics Department, University of KwaZulu-Natal, Durban, South Africa

${ }^{2}$ Abenezer Consulting PLC, Economic Evaluation and Health Care Financing

Division, Addis Ababa, Ethiopia

Full list of author information is available at the end of the article
}

(c) The Author(s). 2019 Open Access This article is distributed under the terms of the Creative Commons Attribution 4.0 International License (http://creativecommons.org/licenses/by/4.0/), which permits unrestricted use, distribution, and reproduction in any medium, provided you give appropriate credit to the original author(s) and the source, provide a link to the Creative Commons license, and indicate if changes were made. The Creative Commons Public Domain Dedication waiver (http://creativecommons.org/publicdomain/zero/1.0/) applies to the data made available in this article, unless otherwise stated. 


\section{Background}

Since the 1980s, HIV/AIDS has struck every aspect of human life. HIV/AIDS has affected the household economy, agricultural productivity, business, education, the health sector and the economic development of countries across the globe. Globally in 2012, 35 (32.2 to 38.8) million people were living with HIV/AIDS, 2.3 (1.9 to 2.7) million people were newly infected with HIV, while 1.6 (1.4 to 1.9) million died of HIV/AIDS [1]. In 2015, $52 \%$ (19 million) of the 36.7 million people with HIV/ AIDS worldwide were living in the eastern- and southern African regions [2]. Since 2010, an increase in the number of people living with HIV, as well as a decrease in new HIV infections and AIDS-related deaths have been reported [1-3]. Infections among children decreased by $35 \%$ in 2012 relative to 2009 [2], even though new HIV infections in children had been projected to account for $13 \%$ of the global total infections during the period 2005-2015 [4].

In Ethiopia, the HIV epidemic has progressed steadily over the last two decades. According to the single point estimate by the Federal HIV/AIDS Prevention and Control Office [5], there were 541,414 new diagnoses of HIV-positive pregnant women and 98,283 HIV-positive births from 2004 to 2010 . In the general population, the past four surveillance rounds indicated the highest HIV prevalence of $5.2 \%$ in urban areas and the lowest of $0.8 \%$ in rural areas [6-8]. Recent HIV prevalence projections (2011-2016) indicate that 2,595,479 patients were in need of antiretroviral therapy (ART) during the period 2011-2016,with 65 and 35\% of ART needs reported in urban and rural settings, respectively [9]. Within the same period, out of a projected total of $25,220,323$ orphans in urban and rural areas, 4,554,568 orphans would be as a result of AIDS. Including the direct vertical transmission of HIV from mother to child, a total of 109,105 new HIV infections was estimated in the country in the period 2011-2016 [9]. Although these projections suggest a high impact of HIV/AIDS in the country overall, it is worth noting that the last four rounds of surveillance and national demographic surveys reports revealed higher HIV prevalence in urban areas than in rural settings $[6-8,10]$. The most recent sentinel surveillance results also showed higher HIV prevalence among pregnant women in the urban antenatal care health facilities [6].

PMTCT interventions have been playing a cross-cutting role, making an impact on three of the previously targeted international Millennium Development Goals (MDGs). These goals were related to the reduction of child mortality, maternal health and HIV/AIDS. PMTCT is also having an effect on the current Sustainable Development Goals (SDG)/ Universal Health Coverage (UHC) targets. Despite their expected role in the international development agenda, PMTCT services have not been widely implemented or utilised by eligible pregnant women [11]. According to the Ethiopian Federal Ministry of Health, in the period 2014-2016, the national uptake of the PMTCT service was 57\% [11]. This was very low as compared to the $90 \%$ coverage in Ghana, Zambia, Namibia and Botswana [1]. In 2012, Ethiopia was also one of the 22 priority countries achieving less than $50 \%$ of antiretroviral treatment coverage for HIV-positive pregnant women [1]. Furthermore, there has been a dearth of costing studies to guide policy decision making in this country [12].

With the elimination of mother-to-child transmission (eMTCT) of HIV infection as a top priority, one piece of crucial information needed by policy-makers and funding organisations concerns the best use of available budgets. Another critical piece of related information includes costing and efficiency analysis for budgeting and cost-effective decisions and the existing literature has not exhaustively analysed these issues [13, 14]. Limited evidence in this respect is likely to lead to inefficient use of resources [12].

Ethiopia, particularly, has faced a dearth of costing evidence to inform PMTCT programme implementation. To the best of our knowledge, Kombe et al. [15], Bikilla et al. [16] and Federal HIV/AIDS Prevention and Control Office (FHAPCO) [17] provide the only studies that report evidence-related to the costs of HIV/AIDS services. Kombe and colleagues [15] surveyed six regional hospitals and estimated the scale-up cost for antiretroviral treatment, voluntary counselling and testing and PMTCT services. Bikilla et al. [16] reported the average cost estimates for the antiretroviral treatment of HIV services at a hospital located in the southern part of the country. More recently, a national antiretroviral treatment costing survey was conducted by FHAPCO [17],although it focused on outpatient costs only, excluding inpatient-related resources. None of these studies addressed the cost of PMTCT services across heterogeneous HIV prevalence contexts (urban versus rural) applying a bottom-up ingredient costing approach. This study aims to fill this gap.

\section{Overview of the PMTCT service}

The government's response to prevent mother-to-child HIV transmission can best be understood within the broad context of the health sector strategy in Ethiopia. The country introduced the Health Sector Development Program IV (HSDP IV) plan as a health chapter of the national Growth and Transformation Plan (GTP) during the period 2011 to 2015 [18] and launched a new Health Sector Transformation Plan (HSTP) for 2015/16-2019/20 [11]. 
The programme started in 2001 at four pilot hospitals in Ethiopia. Thereafter, the intervention was scaled up to 14 health facilities using a single dose Nevirapine (NVP) regimen through a project called HAREG. HAREG was the first pilot project for PMTCT and operated through a close partnership between the FHAPCO and the President's Emergency Plan for AIDS Relief (PEPFAR). The PMTCT service was further integrated with the maternal and child health programmes in 10 hospitals and 13 health centres [19]. Two PMTCT treatment options, single dose NVP and dual antiretroviral (ARV) prophylaxis, were started in 2007. Subsequently, a comprehensive four-pronged PMTCT strategy was adopted $[20,21]$ consisting of prevention of HIV infection; prevention of unintended pregnancy; direct (vertical) transmission prevention of HIV from mothers to infants; and treatment, care and support. The detail of the programme rollout in Ethiopia is summarised in Fig. 1.

PMTCT services were provided during antenatal care (ANC) follow-up and at labour and delivery units as well as during post-delivery care. In accordance with the guideline, all pregnant women coming for ANC visits are requested to agree to HIV pre-testing services through opt-out counselling (allowing them to refuse) $[20,21]$. If the woman agrees, HIV-testing is conducted. If she does not, she will be offered another opportunity to do the test during the next ANC visit. If the pregnant woman is HIV-positive, she will pass through post-test HIV-positive counselling, which includes services such as providing HIV test results and information on the importance of antiretroviral prophylaxis, requesting HIV testing and counselling for the partner and providing relevant information on exclusive breastfeeding. During follow-up visits, the pregnant woman will initiate ARV prophylaxis treatment. A CD4 count will be conducted every six months. Following the national Health Sector Development Plan (HSDP), in 2012 the Ethiopian government launched the Accelerated Elimination Strategic Plan [22]. In the same year, the World Health Organization (WHO) treatment option $\mathrm{B}+$ was adopted [23]. The detail of the programme flow is explained in Fig. 2.

\section{Methods}

The analysis employed a micro-costing ingredient approach from the perspective of the health care provider. According to the micro-costing, bottom-up approach, the following costing procedures were followed: framing the cost analysis; developing the cost inventory; quantifying costs; and calculating summary measures [24]. The data collection commenced in August 2015 and ended in September 2015.

\section{Costing methods}

As suggested by Drummond et al. [25], ingredient items were identified, estimated and valued with their corresponding unit prices. As the first step, resource ingredients, such as health care professionals' time, medical

The Prevention of Mother-to-Child HIV Transmission Program Implementation (2001-2016)

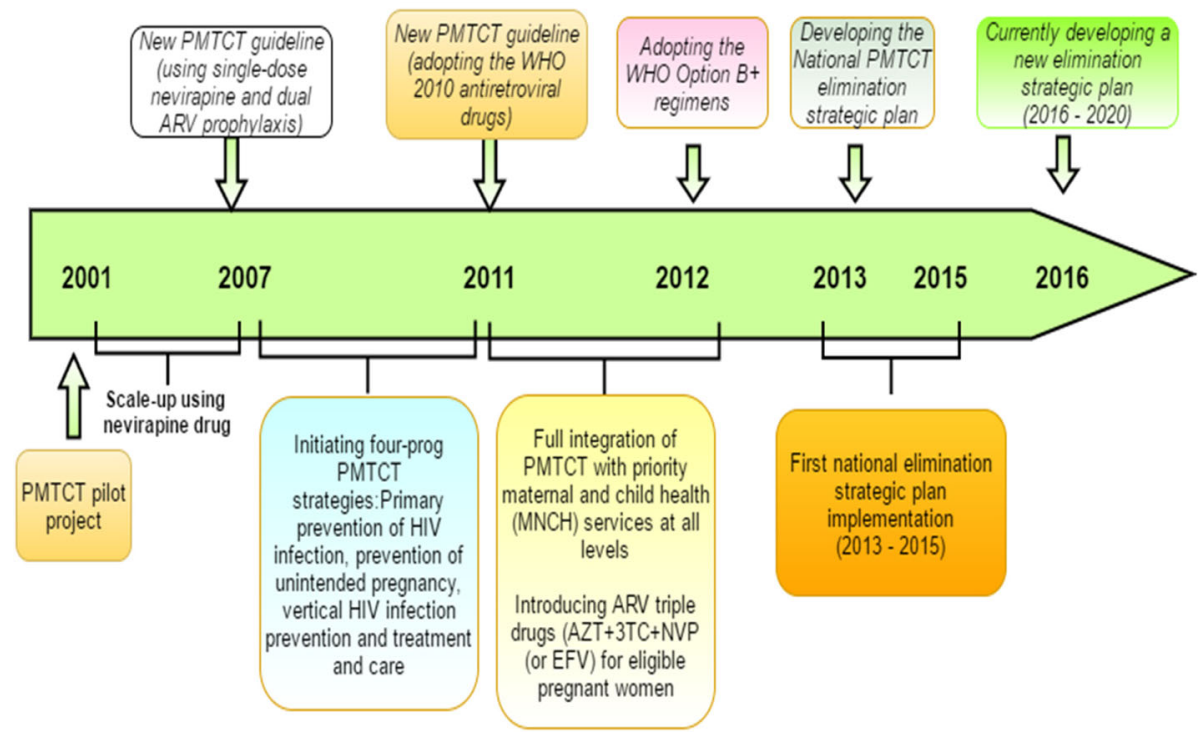

Fig. 1 PMTCT programme implementation (2001-2016) in Ethiopia. Own design 
The Prevention of Mother-to-Child HIV Transmission of HIV Program at the Antenatal care Settings

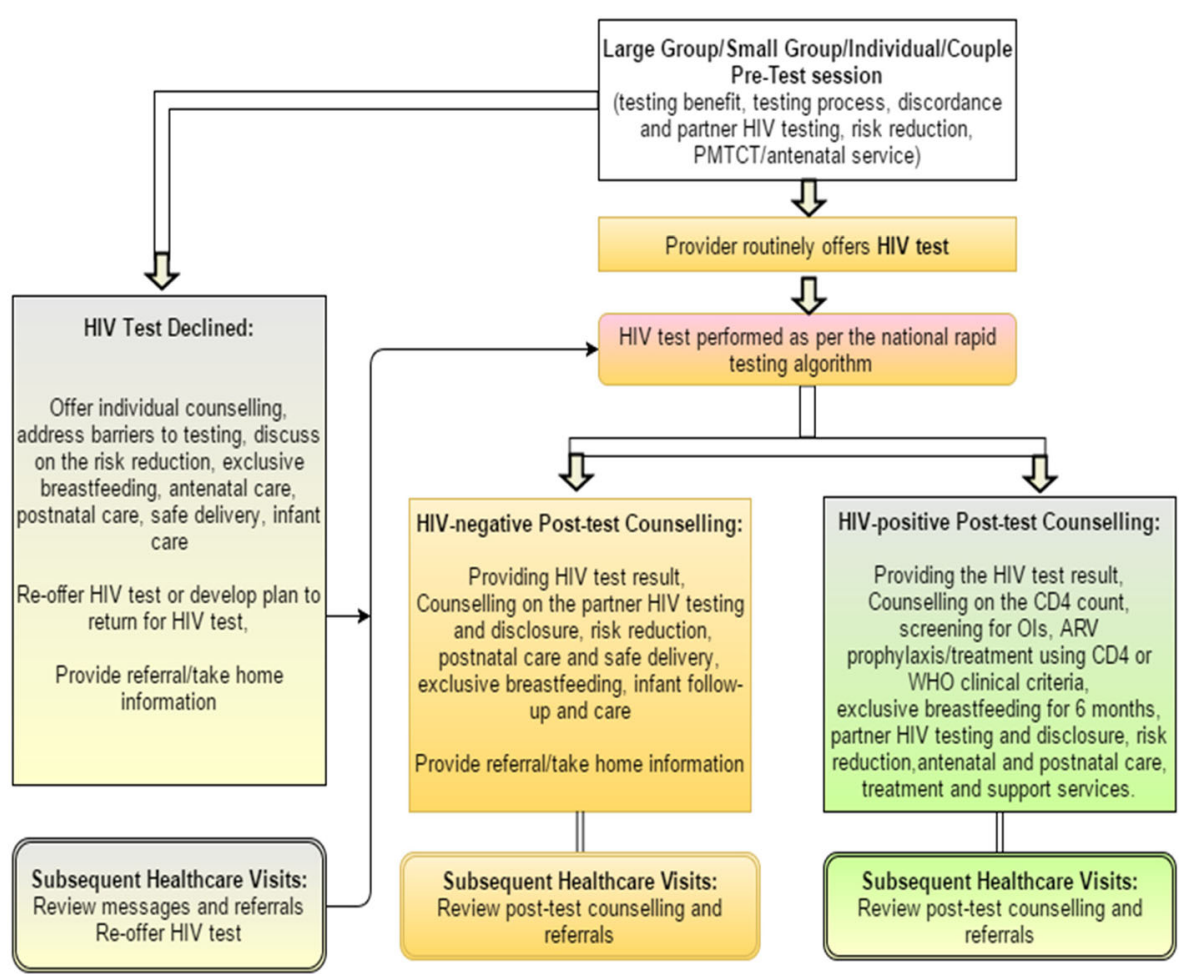

Fig. 2 The PMTCT programme different service packages in Ethiopia. Federal HIV/AIDS prevention and control office PMTCT guideline, 2007 and 2011

supplies, medicines, equipment, transportation, infrastructure and training costing data were identified. Applying an interview costing guide, expert interview sessions were conducted with the PMTCT focal person at the surveyed health facilities to determine yearly quantities utilised. The valuations of resources were estimated considering the average cost data from the Pharmaceutical Fund and Supply Agency (PFSA) in which prices were reported in the 2014 Ethiopian birr (ETB). These prices were converted to the United States dollar (USD) at the 2014 weighted exchange rate (1 US\$ $=19.6705$ ETB) [26].

The costing analysis employed the following formula: $\mathrm{C}=\mathrm{L}+\mathrm{Co}+\mathrm{E}+\mathrm{I}$, where $\mathrm{C}=$ cost, $\mathrm{L}=$ labour, $\mathrm{Co}=$ consumables, $\mathrm{E}=$ equipment and $\mathrm{I}=$ infrastructure. Prices were adjusted for inflation, employing International Monetary Fund (IMF) estimates [27]. An inflation conversion factor and 2014 as a base year were used. Additional costing data, related to the programme management and training costs, were collected at the regional health bureaus. We used a 3\% annual discount rate in accordance with standard practice in the literature [28].

Each resource ingredient (fixed and variable) was measured per pregnant woman-infant pair per year (PPY).
Costing was reported per PPY for urban and rural settings to be able to compare differences across these two settings. In addition, national estimates of the number of HIV-positive pregnant women in need of treatment were based on the Federal Ministry of Health/ Ethiopian Public Health Institute's HIV related estimates and projection database [9]. This number, in turn, was used to estimate the national costs of PMTCT services in rural and urban settings.

\section{Study site selection}

The Ethiopian Public Health Institute (EPHI) identified 117 antenatal-based sentinel surveillance sites in different geographical regions in the country [6]. Of the total 117 ANC sites, six urban health facilities, with the highest HIV prevalence among pregnant women ranging from 8.1 to $17.3 \%$, and six rural health facilities, with a prevalence rate ranging from 0.01 to $0.1 \%$, were selected. Heterogeneity in both HIV prevalence and urban and rural locations was used as criteria to select 12 health facilities, which are located in six regions across Ethiopia. The choice of highest and lowest HIV prevalence health facilities (ANC-PMTCT sites) was informed by the need to obtain a clear picture on differences in costs. Urban areas recorded higher HIV prevalence, which is 
attributed to the fact that urban areas host most-at-risk population groups, notably sex-workers, drug injectors, long-distance truck drivers, members of the armed forces and men who have sex with men. The profile of the 12 health facilities in terms of regional origin, rural and urban context and level of HIV prevalence, is reported in Table 1.

\section{PMTCT service packages}

According to PMTCT service protocols, PMTCT service packages cover HIV pre-test counselling, HIV testing, HIV-negative post-test counselling, HIV-positive posttest counselling, ART (Option $\mathrm{B}+$ ) treatment initiation and counselling, Option $\mathrm{B}+$ follow-up, drug refilling and counselling (for the mother and infant pair), CD4 count (blood sample) service, early infant diagnosis (EID) service, programme management, including the community-based Health Development Army and referral linkage with care and support. Resource ingredients were mapped and estimated for the individual PMTCT service component. Service utilisation data were collected from the Regional Health Bureaus (RHBs) monitoring and evaluation departments. Additional health facility records and performance data were also retrieved from routine Health Management Information System (HMIS) 2013 and 2014 databases. Supplementary national PMTCT service data, such as infrastructure building costs, equipment and medical supplies, were collected from the Federal Ministry of Health $(\mathrm{FMoH})$, the Pharmaceutical Fund and Supply Agency (PFSA) and the FHAPCO.

\section{Average cost analysis}

Detailed average cost analysis steps were conducted as follows:

Labour cost: The national public sector health professionals' salary scale was obtained from the $\mathrm{FMoH}$ Human Resource Development and Management directorate [29]. Each salary category consisted of salary scale levels (from 0 to 10) depending on the years of working experience and education qualification. The median salary scale was calculated for each health care cadre. In addition to the basic salary, additional labour benefit packages, such as housing and night duty allowances were estimated from the expert interview sessions. The net working days was calculated, after deducting annual leave, national days and the sick leave allowance in line with the Ethiopian civil servant protocol (Federal Civil Servant Proclamation, no 262/ 2002 [30]). Staff time spent on PMTCT services was determined by asking team leaders/managers the proportion of time each staff/cadre spends on a specific PMTCT service. The study applied that proportion to the daily rate of that category of staff.

Supplies cost: First, in the surveyed health facilities, a list of relevant supplies used for the PMTCT service was compiled in consultation with health care professionals, including pharmacists and storekeepers. A total of 12 PMTCT focal persons and 10 pharmacists were interviewed to identify the supplies on the list. The complementary unit of the costing measure for identified resources was retrieved from the National Pharmaceutical Fund and Supply Agency (PFSA) national documents for 2014 [23, 24]. For those supply

Table 1 List of costing study sites, including the HIV prevalence and health facility location

\begin{tabular}{llll}
\hline Surveyed health facilities & Region & HIV Prevalence at the health facility (\%) & Direction from centre \\
\hline Bahir Dar Hospital & Amhara & 17.3 & Northern Ethiopia \\
HiwotFana Hospital, & Harrari & 8.8 & Eastern Ethiopia Urban \\
DileChora Hospital & Dire Dawa & 8.1 & Eastern Ethiopia Urban \\
AFRTH Hospital & Addis Ababa & 8.7 & Addis Ababa Urban \\
Soddo Health Center & SNNPR & 8.8 & Southern Ethiopia Urban \\
Teklehaimanot Health Center & Addis Ababa & 8.8 & Addis Ababa Urban \\
Limuseka Health Center & Oromiya & 0.02 & Western Ethiopia \\
Daddim Health Center & Oromiya & 0.04 & Western Ethiopia \\
Toke Health Center, & Oromiya & 0.01 & Western Ethiopia \\
Chewaka Health Center & Oromiya & 0.05 & Western Ethiopia \\
Kokosa Health Center & Oromiya & 0.02 & Eastern Ethiopia \\
Hasange Health Center & Harrari & 0.03 & Eastern Ethiopia \\
\hline
\end{tabular}

Source: Antenatal care (ANC) sentinel HIV/AIDS PMTCT surveillance report [6]: Ethiopia Public Health Institute (EPHI). Report on the 2012 round Antenatal case based sentinel surveillance in Ethiopia. Addis Ababa. Ethiopia; 2014

The HIV prevalence at ANC sentinel surveillance site was measured as the number of women using antenatal service, who are HIV-positive, as a proportion of total number of women who are attending/using antenatal services during the report period (2012) 
items where there were no data in 2014, we collected data from 2011 to 2013 at PFSA [31, 32]. Infrastructure cost: The cost of infrastructure was estimated using the direct allocation approach, based on the average client visits per day [25]. In fact, details of the number of rooms in the health facility, rooms used for PMTCT service, the presence of other integrated services provided at the PMTCT rooms, the average number of clients other than PMTCT clients and the average number of PMTCT clients at the rooms were collected. The information about the type of room, that is, whether the room was jointly used for both PMTCT service and maternal and health services or exclusively dedicated to PMTCT, was ascertained during field data collection. An estimated cost of health facility (hospital and health centres) construction was collected from the FMoH infrastructure directorate. The maintenance and utilities cost was also estimated in consultation with senior experts at the $\mathrm{FMoH}$. The daily average cost of the infrastructure was calculated considering the data inputs: total cost of a building, working life years, annual discount rate, annual equivalent cost, inflation index, expected operation in months or days and average cost per day of operation. Together, the cost of infrastructure, maintenance and utilities per room were apportioned based on the average PMTCT client visits per day.

Equipment cost: The different types of medical equipment (e.g. weighting scale for adults/infants, meters, blood pressure apparatus, stethoscopes, examination beds, chairs, tables, shelves, refrigerators, computers, etc.) used in the PMTCT service were identified, including year of procurement. The unit cost was collected from the PFSA equipment lists. For some of the equipment, where there were no price data from the PFSA, a local market assessment was conducted. The useful life was collected primarily from the Ethiopian government procurement documents. For those items where there was no monetary record, supplementary costing evidence was collected from the World Health Organization Choosing Interventions that are Cost-Effective (WHO-CHOICE) website [33]. For instance, to estimate the average cost of the weighting scale (for the infant), first, the year of purchase was reviewed and, second, the total estimated cost extracted from PFSA were considered. Finally, the annual equivalent cost and a breakdown by the number of days in operation were estimated. The cost of equipment for PMTCT was apportioned based on the number of PMTCT clients.

Training cost: Details of relevant training received by staff were collected at the surveyed health facilities. A total of 12 PMTCT health professionals were interviewed and asked whether they had received
PMTCT training during the past three years since the eMTCT strategy started. If they actually received formal training as per the PMTCT protocol [34] or the current draft guidelines, the total cost of training was collected from the RHBs databases. The annual training cost was calculated based on the number of training participants. The annual training cost was estimated per each health facility. Finally, the average cost of the training (per PPY) was apportioned based on annual PMTCT clients at the health facility.

Programme management: According to the revised PMTCT guidelines and strategic plan documents [34, 35], programme management, monitoring and evaluation were the critical extra-facility inputs for improving quality and service harmonisation across the service package [34]. The amount of time spent and resources used for daily, weekly and quarterly review and for management meetings were collected and estimated at the surveyed health facilities. Additional data including per diem rates, allowances and incentives were also collected. Finally, at each health facility, the annual estimated cost attributed to the programme management was estimated and further apportioned to calculate the average cost per PPY.

CD4 count and EID services were considered in the PMTCT service packages. The different resources for providing CD4 and DBS (dried blood spot) testing were computed by applying a similar costing technique, notably apportioning labour cost based on time spent on providing the test and other shared costs on the basis of patients using CD4 counts. The resource allocated directly to CD4 count testing include the cost of inputs, such as consumables, used in this service.

\section{Sensitivity analysis}

A sensitivity analysis in the estimation of national estimates was conducted using three scenarios: at the 57\% current coverage, at the $75 \%$ coverage level and at $100 \%$ coverage level, although, caveat needs to be born in mind. The assumption of $100 \%$ coverage level might result in more than the costs obtained in sensitivity analyses due to possible diseconomies of scale that might arise from managing a large-scale response of such a magnitude.

\section{Ethical considerations}

The research project was submitted and approved by the University of KwaZulu-Natal (UKZN) Biomedical Research and Ethics Committee (BREC REF 385/14) and the Federal Ministry of Health/ Ethiopian Public Health Institute (EPHI) Scientific and Ethical Review Office (SERO REF: 6.13/80). The data collection tool and Excel costing sheet were adapted from a similar costing survey 
in South Africa and from a malaria elimination costing sheet [36]. To refine the tool, it was piloted in two health facilities in Addis Ababa: Gandhi Memorial Hospital and Beletshachew Health Center.

\section{Results}

In urban high HIV prevalence health facilities, the estimated cost of providing a PMTCT service per PPY ranged from ETB 6280 (USD 319) to ETB 21,620 (USD 1099). The mean USD per PPY was higher in hospitals than in health centres. The mean cost of providing a PMTCT service (per PPY) was ETB 13,782 (USD 701) with the highest cost of ETB 20,779 (USD 1056) and ETB 21,620 (USD 1099) recorded at HiwotFana Hospital and AFRTH hospital, respectively. In rurally located low HIV prevalence health facilities, on the other hand, the average cost per PPY ranged from ETB 4323 (USD 220) to ETB 7539 (USD 383) with a mean cost per PPY of ETB 5935 (USD 302). The cost variation was explained by the type and quantity of resource ingredients and by the type of health facilities (health centre, hospital centre). Most of the facilities in urban high HIV prevalence settings were hospitals (including HiwotFana and AFRTH), which were better equipped with different resources (labour, supplies and capital) relative to health facilities typically in rural settings. The detailed average cost per each health facility is described in Table 2. The details with respect to each resource input are explained in Table 3.

\section{Cost components}

In urban high HIV prevalence settings, the infrastructure cost accounted for the highest proportion of the total cost (48\%) followed by labour (26\%) and consumables (22\%), while the consumables cost accounted for the highest proportion (42\%) in rural low HIV prevalence health facilities followed by infrastructure and labour cost, which accounted for 26 and $20 \%$ of the estimated cost, respectively.
Of the listed consumables in rural areas, antiretroviral drugs and other drugs costs, including costs of Zidovudine (AZT) + Lamivudine (3TC) + Nevirapine (NVP), Isonicotinylhydrazide (INH) prophylaxis, cotrimoxazole, anti-bacterial drugs, NVP prophylaxis syrup and cotrimoxazole syrup (for the infants), comprised more than $80 \%$ of the total cost. The cost of labour does not rank second in rural area as it does in urban areas due to understaffing and relatively junior health care workers (who had a diploma or lower qualification) in rural setting.

Overall, the costs per PPY are higher in urban settings. Higher costs per PPY in urban settings are explained by first, higher cost of infrastructure, equipment and labour in urban areas and; secondly, the higher number of visits in urban areas as compared to the rural areas. Furthermore, the estimation of the average cost per PPY and not per visit implies higher cost per PPY in urban facilities, where relatively more frequent visits were recorded. The proportion of infrastructure cost varied from 25 to $60 \%$ in urban high HIV prevalence settings.

In both settings (urban high HIV prevalence and rural low HIV prevalence), the lowest proportion of costs (2\%) was incurred for the training. According to the health workers interview report, there were health facilities that did not receive any formal PMTCT service training, or the trained staff had resigned and left the health facility. On the other hand, three resource items, namely consumables, labour and infrastructure contributed to more than $87 \%$ of the unit cost (per PPY). The detailed average cost estimation of the different PMTCT resource ingredients across the urban high HIV prevalence and rural low HIV prevalence settings is summarised in Table 3.

Labour and infrastructure costs accounted for $74 \%$ of the resources used for the PMTCT service provision. This can be explained by the higher cost of infrastructure such as buildings and their maintenance, in addition to relatively expensive experienced experts with many years of working experience, mainly in urban settings.

Table 2 Annual average cost per PPY in urban and rural surveyed health facilities

\begin{tabular}{llllll}
\hline Urban health facilities & $\begin{array}{l}\text { Average cost PPY } \\
\text { (ETB) }\end{array}$ & $\begin{array}{l}\text { Average cost PPY } \\
\text { (USD) }\end{array}$ & Rural health facilities & $\begin{array}{l}\text { Average cost PPY } \\
\text { (ETB) }\end{array}$ & $\begin{array}{l}\text { Average cost PPY } \\
\text { (USD) }\end{array}$ \\
\hline Teklehaimanot Health Center & 6280 & 319 & Atinago Health Center & 5730 & 291 \\
Soddo Health Center & 8407 & 427 & Chewaka Health Center & 4322 & 220 \\
HiwotFana Hospital & 20,779 & 1056 & Dedo Health Center & 7539 & 383 \\
DileChora Hospital & 14,410 & 733 & Hasange Health Center & 6319 & 321 \\
Bahir Dar Hospital & 11,195 & 569 & Kokossa Health Center & 6104 & 310 \\
AFRTH Hospital & 21,620 & 1099 & Toke Health Center & 5598 & 285 \\
Mean & 13,782 & 701 & Mean & 5935 & 302 \\
Stdev (SD) & 6366 & 324 & Stdev (SD) & 1049 & 53 \\
\hline
\end{tabular}




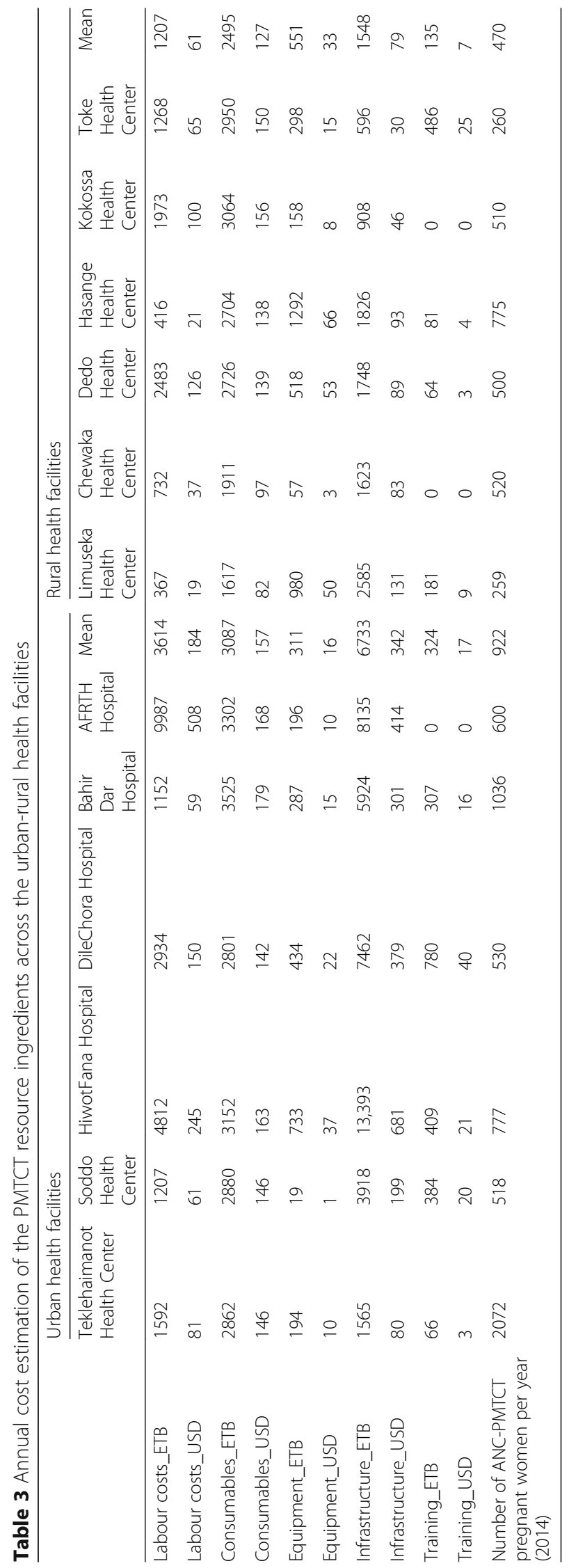


The smallest proportion of the costs was estimated for equipment and training. The list of relevant resources (and calculated cost) used in the hospital and health centres is detailed in Additional files 1 and 2.

\section{Costing the PMTCT service packages}

In the PMTCT service continuum, HIV/AIDS counselling and testing contributed 15 and 10\% in urban high HIV prevalence and rural low HIV prevalence health facilities settings, respectively. Of the listed HIV/AIDS counselling and testing activities, 40 to $50 \%$ of this cost was spent on HIV testing and HIV-positive post-test counselling in each setting. The cost of HIV-negative post-test counselling accounted for 2 to $3 \%$ of the total cost (per PPY) in each setting. As shown in Fig. 3, antiretroviral treatment (option $\mathrm{B}+$ ) follow-up and counselling contributed to the highest proportion of the cost (62\% in urban high HIV prevalence health facilities and $71 \%$ in rural low HIV prevalence health facilities). The major component of these costs was the provision of antiretroviral treatment (option $\mathrm{B}+$ drugs) for the mothers and infants, which constituted 50\%of these costs in rural health facilities and 30\% in urban health facilities. The higher cost is because the provision of ART treatment requires more frequent patient visits to the health facilities and thus more inputs. The CD4 count service and EID accounted for 8 and $4 \%$ of the average cost per PPY in urban and rural settings, respectively.

Programme management accounted for 3\% of the unit cost (per PPY) in the urban high HIV prevalence facilities settings, but was a relatively lower proportion of the cost in rural low HIV prevalence facilities (i.e. 0.5\%). The cost difference is due to the cost incurred by the top management teams at urban facilities for conducting service planning, monitoring and evaluation, as well as for frequent review meetings to improve service quality and efficiency.

\section{National estimates}

At the current coverage level, the total cost of the PMTCT service at the base year, 2014, was estimated to be USD 6 million in urban settings. The corresponding cost for rural settings was estimated to be USD 3 million (Fig. 4). At $75 \%$ coverage, the national estimated total cost was USD 8 million and USD 4 million in urban and

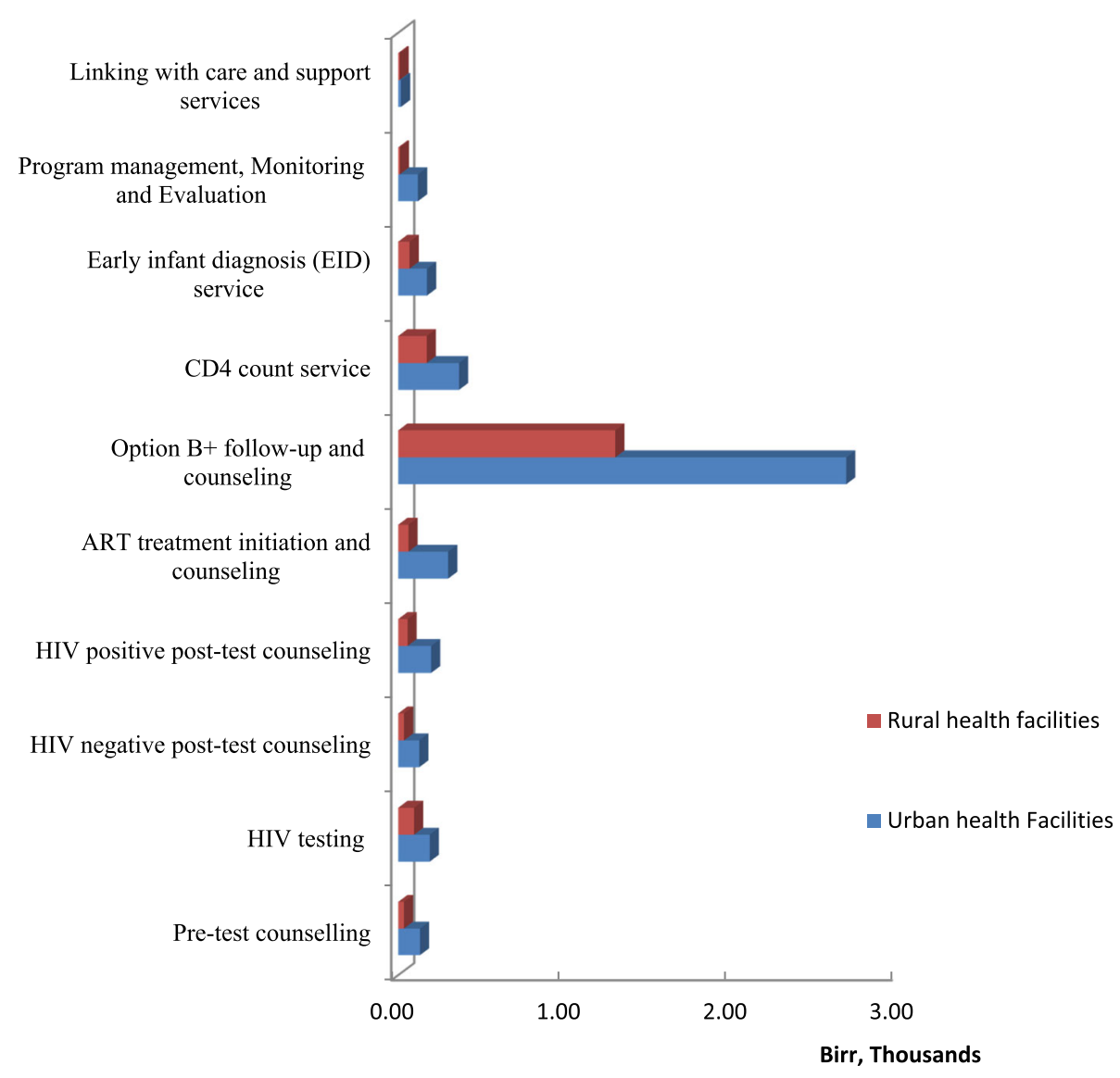

Fig. 3 Cost of PMTCT service components in urban and rural health facilities 


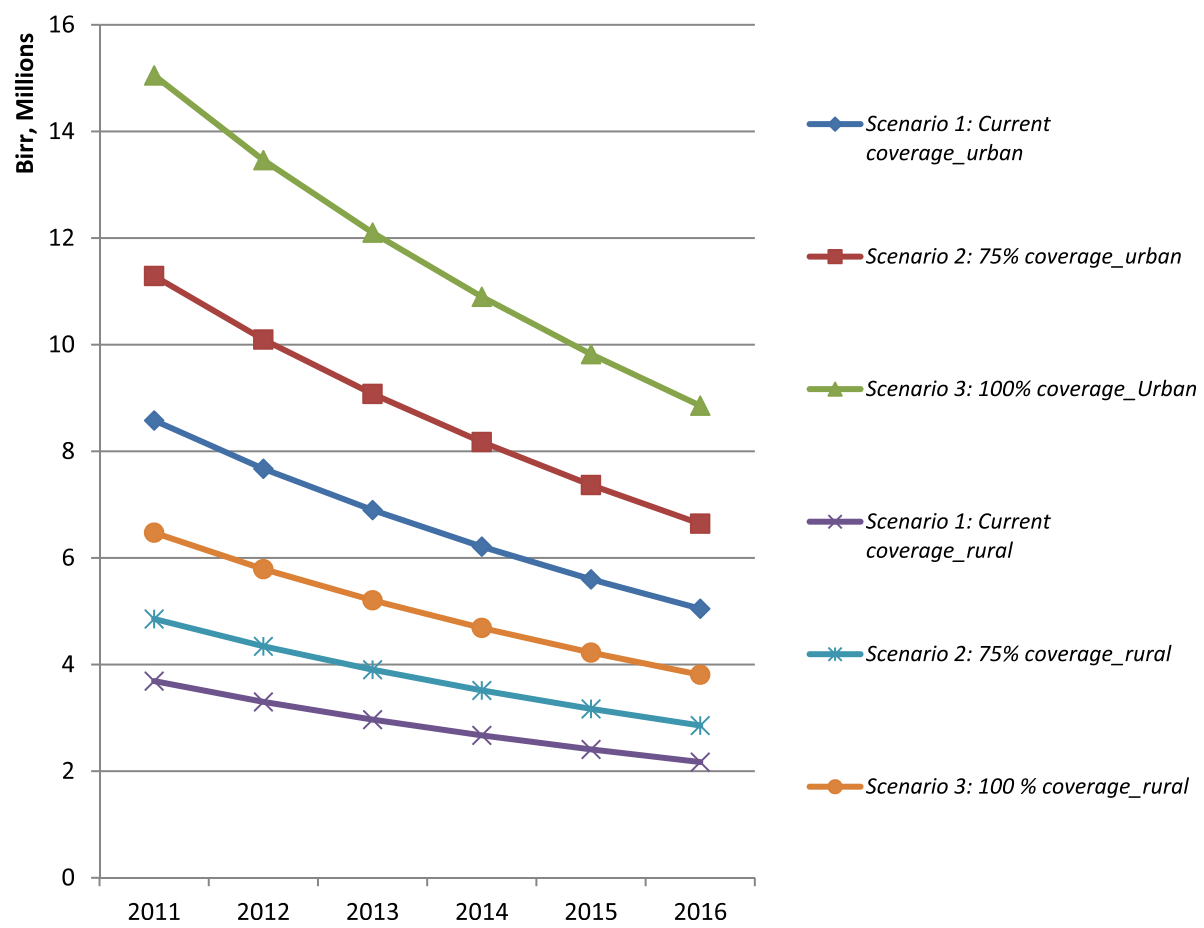

Fig. 4 The national PMTCT service cost estimation in urban and rural settings, 2011 to 2016

rural settings, respectively. If the service access improved to $100 \%$ universal coverage, the total cost of the service in urban areas would increase to USD 11 million, while the cost in rural areas would increase to USD 5 million. A caveat needs to be borne in mind here that the cost of $100 \%$ universal coverage could be higher due to diseconomies of scale that might arise from difficulties in implementation and management of the programme.

\section{Discussion}

While local context costing evidence is relevant for healthcare planning, budgeting and cost-effectiveness analysis, it continues to be scarce in Ethiopia. This study was undertaken to conduct a costing exercises to fill the evidence gap by distinguishing the costing evidence of PMTCT in rural and urban settings. The costing analysis indicated that cost per PPY ranged from 4323 ETB (USD 220) in low HIV prevalence rural health facilities to 21,620 ETB (USD 1099) in high HIV prevalence urban health facilities. Estimates from similar studies in sub-Saharan African countries reported comparable average costs per PPY. A study by Touréet al. [37], for example, reported unit costs in the range of USD 203 to USD 1030 in Namibia and USD 94 to USD 342 in Rwanda. The studies in Namibia and Rwanda both applied an ingredient-based costing including HIV testing and counselling, CD4 cell count service, antiretroviral drugs, community-based activities, provision of medication postpartum, co-trimoxazole with prophylaxis and
EID. In Rwanda, none of the surveyed health facilities had a CD4 count service, therefore, this was not captured in the costing estimation, while in Namibia the facilities costing included a CD4 count service. In addition, wages in Namibia were higher than in Rwanda. Similarly, our cost estimate in urban health facilities ranged from USD 319 to USD 1099.12. According to our analysis, urban health facilities were better equipped and resourced than rural facilities, which may be similar to Namibia's health facilities. However, a relatively low unit cost was estimated in rural low HIV prevalence areas (USD 220 to USD 383), which is more comparable with the unit costs reported in Rwanda. In Ethiopia, Bikilla et al. [16] reported a unit cost (per PPY) of USD 235 and USD 29 for ART outpatient and inpatient care, respectively. This finding was also comparable to the low HIV prevalence rural health facilities costing report, which ranged from ETB 4323 (USD 220) to ETB 7539 (USD 383).

Our analysis also unveiled a wide variation of costing estimates between urban and rural settings, which was explained by the difference in the type and quantity of resources (labour, consumables and infrastructure) used across the high and low HIV prevalence and urban-rural settings, as well as the type of health facilities sampled for the costing analysis. Most of the facilities in urban high HIV prevalence settings were tertiary level hospitals (four out of the six health facilities) that were better equipped in terms of resources as compared to the rural 
health facilities that were less equipped in terms of labour, equipment and infrastructure. The higher costs of infrastructure and other inputs into PMTCT services in urban settings meant that more patients' visits resulted in no reduction of cost per PPY and this yielded no economies of scale. In fact, previous studies have also used the same argument to explain the cost differences. Desmond et al. [36], for example, reported that HIV prevalence and resource distribution were the main determining factors affecting the wide cost variation across well-resourced sites in Western Cape and Limpopo provinces and low HIV prevalence and poorly-resourced provinces in KwaZulu-Natal and Free State provinces in South Africa. In another study, Desmond and Boyce [38] also assessed the financial and economic cost differences in the rural PMTCT sites in Eastern Cape, South Africa and found that staff time was the main cost contributor. However, our study identified medical consumables (in rural low HIV prevalence sites) as the main cost driver. The difference was because our study considered the new PMTCT Option B+ lifelong treatment, while the study by Desmond and Boyce [30] considered the single-dose nevirapine and cotrimoxazole antiretroviral prophylaxis drug regimens.

A literature review by Touréet al. [37] reported 6 to $15 \%$ of the estimated cost to be attributed to the CD4 count service. Similarly, our analysis estimated $8 \%$ of the unit cost (per PPY) as attributable to the CD4 counts-related service. While this study reports similar costing results for some of the PMTCT service packages, it also points out a higher unit cost estimate for infrastructure that comprised $48 \%$ of the unit cost of the PMTCT service (per PPY). This may be attributed to the low PMTCT service uptake and underutilisation of the service by antenatal care-attending HIV-positive pregnant women in the country [1, 11]. According to UNAIDS [1], Ethiopia is listed as one of the 22 targeted countries achieving less than 50\% coverage in 2012, which, as already noted, is very low as compared to $90 \%$ coverage in Ghana, Zambia, Namibia and Botswana. For instance, the 2011/12 Ministry of Health Annual Performance Report [39] indicated that a total of 9775 HIV-positive mothers received ART treatment in the 2004 Ethiopian fiscal year (EFY), which is far below the target set for the year (i.e. 38,405).

In the current effort to eliminate new HIV infections and to support the current UHC agenda, the relevance of having context-specific costing data for PMTCT and analysing the differences between urban and rural health facilities is critical. However, Ethiopia in particular, has faced a lack of critical evidence on the cost of PMTCT programmes [15-17]. Earlier costing studies have analysed the cost and financial requirements for the scale-up of HIV/AIDS services in limited parts of the country. However, neither of these studies assessed the PMTCT service taking into account HIV heterogeneity across urban-rural health facilities, which is believed to be important for improving health care delivery efficiency [40, 41], performance management [42], future improvement opportunities [40] and identifying the potential for cost containment and reduction [43]. This study contributes to the literature by providing this evidence.

Although the analyses highlighted the unit cost and national costing difference in urban high HIV prevalence versus rural low HIV prevalence settings, the study has some important shortcomings. The reported unit cost results do not necessarily reflect the level of efficiency, as the outcome data over a period of time was not analysed. This study only provided a snapshot of costing results across the heterogeneous HIV prevalence and urban-rural settings. However, considering these findings and complementary panel data, future research may report the technical or allocative efficiency differences across these settings. In addition, we considered the estimated unit cost result per PPY from the two settings (urban high HIV prevalence and rural low HIV prevalence) to estimate the national PMTCT service cost from the health care provider perspective. However, the facilities were not randomly selected and costing estimates derived from nationally representative sampled health facilities might be required.

\section{Conclusions}

The current global economic downturn and reduction in international finance requires substantial costing evidence to inform health care policy and programme management decisions. This information is particularly critical in countries with heterogeneous HIV prevalence countries, such as Ethiopia. Following a comprehensive costing analysis in this study, the finding is that resources used for PMTCT service packages varied across health facilities and HIV prevalence contexts. Furthermore, providing PMTCT service in the high HIV prevalence, urban health facilities costs more than in the rural facilities. Context-specific costing appears vital to provide locally sensitive evidence for health service management and priority setting.

\section{Additional files}

Additional file 1: Health center resource lists and computed average unit/ annual cost across the different inputs in Ethiopia (DOCX $23 \mathrm{~kb}$ )

Additional file 2: Hospital resource lists and computed average unit/ annual cost across the different inputs in Ethiopia (DOCX 25 kb)

\section{Abbreviations}

AFRTH: Armed Forces Referral and Teaching Hospital; ANC: Antenatal care; ART: Antiretroviral therapy; DBS: Dried blood spot; EFY: Ethiopian fiscal year; 
EID: Early infant diagnosis; eMTCT: Elimination of mother-to-child transmission; EPHI: Ethiopian Public Health Institute; ETB: Ethiopian birr; FHAPCO: Federal HIV/AIDS Prevention and Control Office; FMoH: Federal Ministry of Health; HMIS: Health Management Information System; IMF: International Monitoring Fund; MDGs: Millennium development goals; NVP: Nevirapine; PFSA: Pharmaceutical Fund and Supply Agency; PMTCT: Prevention of mother-to-child transmission of HIV/AIDS; PPY: Per pregnant woman-infant pair per year; RHBs: Regional Health Bureaus; SDG: Sustainable development goals; SPSS: Statistical Package for the Social Sciences; UHC: Universal health coverage; UKZN: University of KwaZulu-Natal; USD: United States dollar; WHO-CHOICE : World Health Organization Choosing Interventions that are Cost-Effective

\section{Acknowledgments}

We are grateful to the University of KwaZulu-Natal (UKZN), Health Economics and HIV/AIDS Research Division (HEARD) for its stipend support and to the Ethiopian Public Health Institute (EPHI) for its technical assistance. These institutions did not have any influence on the study design preparation, data collection, analyses or manuscript preparation. The Federal Ministry of Health (FMOH), Ethiopian Public Health Institute (EPHI), Federal HIV/AIDS Prevention and Control Office (FHAPCO), Addis Ababa City Administration Health Bureau, Dire Dawa City Administration Health Bureau, Amhara Regional Health Bureau, Oromiya Regional Health Bureau, SNNP Regional Health Bureau and Harrari Regional Health Bureau facilitated the data collection and provided the required evidence. We would like to extend our gratitude to Sisay Shine for overseeing and supervising the data collection. Finally, we acknowledge the prompt and efficient English editing service from Linda Scott.

\section{Funding}

This work was supported by an African Doctoral Dissertation Research Fellowship award offered by the African Population and Health Research Centre (APHRC) in partnership with the International Development Research Center (IDRC). The funding organization has no role in the study design, analysis, and interpretation of data and in writing the manuscript.

\section{Availability of data and materials}

The detailed spread sheets and materials supporting the study findings were deposited at the EPHI national research database, which may be accessed on request. In addition, the detailed findings were submitted to the School of Accounting, Economics and Finance at UKZN as a requirement for the postgraduate study programme. The data are available and accessible from one of the co-investigators, Elias Asfaw, who can be contacted at abenezerconsultancy@gmail.com.

\section{Authors' contributions}

EAZ designed the study protocol, prepared the data collection tools, developed the costing framework, led the data collection, analysed the costing data, and drafted and revised the manuscript; JM oversaw the data collection, commented on the costing analysis plan, and reviewed and revised the article; SK oversaw the data collection, commented on the costing analysis plan, and reviewed and revised the article. BJ commented on the overall costing method and revised the article. All authors read and approved the final manuscript.

\section{Ethics approval and consent to participate}

The research project was approved by the University of KwaZulu-Natal (UKZN) Biomedical Research and Ethics Committee (BREC REF 385/14) and the Federal Ministry of Health/ Ethiopian Public Health Institute (EPHI) Scientific and Ethical Review Office (SERO REF: 6.13/80). Informed written consents were obtained from the health professionals who were working at the health facilities, the maternal and childcare service head and the antenatal care PMTCT service coordinators.

\section{Consent for publication}

Consents for publication were received from the health professionals who were working at the health facilities, the maternal and childcare service head and the antenatal care PMTCT service coordinators.

\section{Competing interests}

The authors declare that they have no competing interests.

\section{Publisher's Note}

Springer Nature remains neutral with regard to jurisdictional claims in published maps and institutional affiliations.

\section{Author details}

${ }^{1}$ Economics Department, University of KwaZulu-Natal, Durban, South Africa.

${ }^{2}$ Abenezer Consulting PLC, Economic Evaluation and Health Care Financing Division, Addis Ababa, Ethiopia. ${ }^{3}$ Public Administration and Economics Department, Durban University of Technology, Durban, South Africa. ${ }^{4}$ Abt Associates Inc, Bethesda, USA.

Received: 9 February 2018 Accepted: 27 February 2019 Published online: 06 March 2019

\section{References}

1. UNAIDS, Global report. UNAIDS report on the global AIDS epidemic 2013. Joint United Nations Programme on HIV/AIDS. Switzerland: United Nations Programme on HIV and AIDS; 2013.

2. UNAIDS, Global report. UNAIDS report on the global AIDS epidemic 2016. Joint United Nations Programme on HIV/AIDS. Switzerland: United Nations Programme on HIV and AIDS; 2016.

3. UNAIDS, Global report. UNAIDS report on the global AIDS epidemic 2012. Joint United Nations Programme on HIV/AIDS. Switzerland: United Nations Programme on HIV and AIDS; 2012.

4. Stover J, Bertozzi S, Gutierrez JP, Walker N, Stanecki KA, Greener R, et al. The global impact of scaling up HIV/AIDS preventions in low and middle income countries (LMICS). American association for the. Advancement of Science. 2006;311(5766):1474-6.

5. FHAPCO. Single Point HIV Prevalence Estimate. In: Federal HIV/AIDS Prevention and Control Office. Addis Ababa. Ethiopia: Federal Ministry of Health; 2007.

6. EPHI. Report on the 2012 round antenatal case based sentinel surveillance in Ethiopia: Ethiopian Public Health Institute. Addis Ababa. Ethiopia; 2014.

7. HAPCO \& GAMET. HIV/AIDS in Ethiopia: an epidemiological synthesis. In: Ethiopia HIV/AIDS Prevention \& Control Office (HAPCO) and global AIDS monitoring \& evaluation team (GAMET) and World Bank global HIV/AIDS program; 2008.

8. FHAPCO. HIV/AIDS in Ethiopia an Epidemiological Synthesis. Federal HIV/ AIDS Prevention and Control Office. Addis Ababa, Ethiopia; 2013.

9. EHNRI. HIV Related Estimates and Projections for Ethiopia. Addis Ababa, Ethiopia: Ethiopian Health and Nutrition Research Institute, Federal Ministry of Health; 2012.

10. ECSA. National Ethiopia Demographic Health Survey (E-DHS) 2011 report. Ethiopia Central Statistical Agency, Addis Ababa, Ethiopia; 2011.

11. FMOH. Health Sector Transformation Plan (HSTP) 2015/16-2019/20. Addis Ababa, Ethiopia: Federal Ministry of Health; 2015.

12. Beck EJ, Avila C, Gerbase S, Harling G, De Lay P. Counting the cost of not costing HIV health facilities accurately: pay now, or pay more later. PharmacoEconomics. 2012;30(10):887-902.

13. Ades AE, Ratcliffe J, Gibb DM, Sculpher MJ. Economic issues in the prevention of vertical transmission of HIV. Pharmacoeconomics. 2000;18:912.

14. Galárraga O, Colchero MA, Wamai RG, Bertozzi SM. HIV prevention costeffectiveness: a systematic review. BMC Public Health. 2009. https://doi.org/ 10.1186/1471-2458-9-S1-S5.

15. Kombe G, Galaty D, Gadhia R, Decker C. Human and financial resource requirements for scaling up HIV/AIDS services in Ethiopia. Bethesda, MD: The Partners for Health Reform plus project, Abt Associates Inc; 2004.

16. Bikilla AD, Jerene D, Robberstad B, Lindtjorn B. Cost estimates of HIV care and treatment with and without anti-retroviral therapy at Arba Minch hospital in southern Ethiopia. Cost Effectiveness and Resource Allocation. 2009;7(6):1-7.

17. FHAPCO. Facility-Based Anti-Retroviral Treatment Costing Study in Ethiopia. Addis Ababa, Ethiopia: Federal HIV/AIDS Prevention and Control Office, Federal Ministry of Health; 2012.

18. MOFED. The Federal Democratic Republic of Ethiopia: Growth and Transformation Plan (GTP) 2010/11-2014/15. Addis Ababa, Ethiopia: Ministry of Finance and Economic Development; 2010.

19. AED-LINKAGES. Prevention of Mother to Child Transmission (PMTCT). Addis Ababa, Ethiopia: Baseline Survey, Hareg Project. 
20. FHAPCO. Guidelines For Prevention of Mother-to-Child Transmission of HIV in Ethiopia. Federal HIV/AIDS Prevention and Control Office, Addis Ababa, Ethiopia; 2007.

21. FMOH. Guidline for Prevention of Mother-to-Child Transmission of HIV in Ethiopia. Addis Ababa, Ethiopia: Federal Ministry of Health; 2011.

22. $\mathrm{FMOH}$. The National Strategic Plan for Elimination of Mother to Child Transmission of HIV (e-MTCT of HIV) 2013-2015. Addis Ababa. Ethiopia: Federal Ministry of Health.

23. WHO. Programmatic update: Use of Antiretroviral Drugs for Treating Pregnant Women and Preventing HIV Infection in Infants. Geneva: HIV/AIDS Programme: World Health Organization; 2012.

24. Haddix AC, Teutsch SM, Corso PS. Prevention effectiveness: a guide to decision analysis and economic evaluation. 3nd ed: Oxford University Press; 2003.

25. Drummond MF, Sculpher MJ, Torrance GW, O'Brien BJ, Stoddart GL. Methods for the economic evaluation of health care programs. 3rd ed. New York: Oxford University Press; 2005.

26. Oanda.Historical Exchange Rates. Exchange rate - ETB/USD.http://www. oanda.com/currency/historical-rates/. Accessed 05 Nov 2015.

27. IMF. International Monetary Fund, World Economic Outlook Database April 2014.http://www.imf.org/external/pubs/ft/weo/2014/01/weodata/index.aspx. Accessed 05 Nov 2015

28. Weinstein MC, Siegel JE, Gold MR, Kamlet MS, Russel LB. Recommendations of the panel on cost effectiveness in health and medicine, consensus statement. J Am Med Assoc. 1996;276(15):1253-8.

29. FMOH. Federal Ministry of Health Human resource development and management directorate, manuals and incentive package directive document 200-8/3/6. Addis Ababa: Ethiopia; 2014.

30. FDRE. Federal Negarit Gazeita of the Federal Democratic Republic of Ethiopia, Federal Civil Servants Proclamation No. 262/2002, Addis Ababa, Ethiopia; 2002.

31. FMOH/ PFSA. Report on National Quantification of ARVs, Ol drugs, Laboratory Commodities, medicines for Opportunistic Infections and Sexually Transmitted Infections, Nutrition supplements and Condoms for the years 2015 and 2016. Addis Ababa, Ethiopia: Federal Ministry of Health/ Pharmaceuticals Fund and Supply Agency, HIV/AIDS Prevention and Control office; 2014.

32. FMOH/ PFSA. Report on quantification review exercise of ARVs, Ol drugs, and RTKs for the Federal Democratic Republic of Ethiopia: January 2013 to December 2014, Federal Ministry of Health, Pharmaceuticals Fund and Supply Agency, Federal HIV/AIDS Prevention and Control Program, Addis Ababa, Ethiopia; 2012

33. WHO. World Health Organization, Choosing Interventions that are Cost Effective (WHO-CHOICE), Geneva. https://www.who.int/choice/costeffectiveness/en/. Accessed 16 May 2014.

34. FHAPCO. Guidelines For Prevention of Mother-to-Child Transmission of HIV and Antiretroviral Treatment guidline In Ethiopia. Addis Ababa. Ethiopia: Federal HIV/AIDS Prevention and Control Office; 2008.

35. FMOH. The National Health Sector Development Programme (HSDP IV) 2010/11-2014/15. Addis Ababa. Ethiopia: Federal Ministry of Health; 2010.

36. Desmond C, Franklin L, Steinberg M. The prevention of mother-to-child HIV transmission: costing the service in four sites in South Africa. Health Systems Trust. 2004.

37. Touré $H$, Audibert M, Doughty $P$, Tsague L, Mugwaneza P, Nyankesha E, et al. Public sector services for the prevention of mother-to-child transmission of HIV infection: a micro-costing survey in Namibia and Rwanda. World Health Organization. 2013;91(6):407-15.

38. Desmond C, Boyce G. Assessing the costs of a rural PMTCT pilot sites in the eastern cape. Human sciences research council. Cape Town: south. Africa. 2004.

39. FMOH. Annual Performance Report 2011/12. Addis Ababa, Ethiopia; 2012: Federal Ministry of Health.

40. Ramsey RH. Activity-based costing for hospitals. Hospital \& Health Services Administration. 1994:39(3):385.

41. Kernick D. Costing in primary care--is the truth out there? Fam Pract. 2005; 22(3):225-6.

42. Kernick D. Costing interventions in primary care. Fam Pract. 2000;17(1):66.

43. Ross TK. Analyzing health care operations using ABC. J Health Care Finance. 2003;30(3):1-20

\section{Ready to submit your research? Choose BMC and benefit from:}

- fast, convenient online submission

- thorough peer review by experienced researchers in your field

- rapid publication on acceptance

- support for research data, including large and complex data types

- gold Open Access which fosters wider collaboration and increased citations

- maximum visibility for your research: over $100 \mathrm{M}$ website views per year

At $\mathrm{BMC}$, research is always in progress.

Learn more biomedcentral.com/submissions 